Innovationskompetenz im demografischen Wandel 
Thomas Langhoff - Manfred Bornewasser • Eckhard Heidling • Bernd Kriegesmann • Michael Falkenstein (Hrsg.)

\section{Innovationskompetenz im demografischen Wandel}

Konzepte und Lösungen für die unternehmerische Praxis

Springer Gabler 


\section{Herausgeber}

Professor Dr. Thomas Langhoff

Prospektiv Gesellschaft für betriebliche

Zukunftsgestaltungen $\mathrm{mbH}$

Dortmund, Deutschland

Dr. Eckhard Heidling

Institut für Sozialwissenschaftliche

Forschung e.V. (ISF München)

München, Deutschland

Professor Dr. Michael Falkenstein

Leibniz-Institut für Arbeitsforschung

an der TU Dortmund (IfADo)

Dortmund, Deutschland
Professor Dr. Manfred Bornewasser

Lehrstuhl für Sozialpsychologie,

Arbeits- und Organisationspsychologie,

Universität Greifswald,

Greifswald, Deutschland

Professor Dr. Bernd Kriegesmann

Institut für angewandte Innovations-

forschung e.V. (iAi Bochum)

Bochum, Deutschland

Die dieser Publikation zugrunde liegenden Vorhaben wurden mit Mitteln des Bundesministeriums für Bildung und Forschung (derobino FKZ: 01HH11007 - 01HH11010; IBU FKZ: 01HH11004 - 11006; InnoFaktor FKZ: 01HH11014, 01HH12001; INNOKAT FKZ: 01HH11001 - 11003; KreaRe FKZ: 01HH11011-12) sowie mit Mitteln des Europäischen Sozialfonds gefördert. Die Verantwortung für den Inhalt dieser Veröffentlichung liegt bei den Autoren.

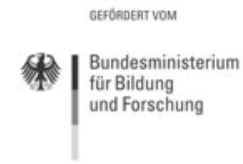

Förderschwerpunkt

Innovationsfähigkeit im demografischen Wandel
ISBN 978-3-658-09158-3

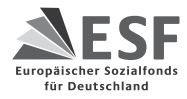

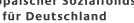

EUROPÄISCHE UNION
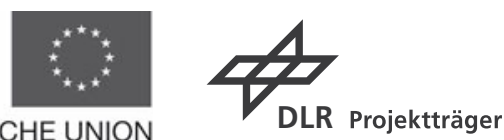

DOI 10.1007/978-3-658-09159-0

ISBN 978-3-658-09159-0 (eBook)

Die Deutsche Nationalbibliothek verzeichnet diese Publikation in der Deutschen Nationalbibliografie; detaillierte bibliografische Daten sind im Internet über http://dnb.d-nb.de abrufbar.

\section{Springer Gabler}

(C) Springer Fachmedien Wiesbaden 2015

Das Werk einschließlich aller seiner Teile ist urheberrechtlich geschützt. Jede Verwertung, die nicht ausdrücklich vom Urheberrechtsgesetz zugelassen ist, bedarf der vorherigen Zustimmung des Verlags. Das gilt insbesondere für Vervielfältigungen, Bearbeitungen, Übersetzungen, Mikroverfilmungen und die Einspeicherung und Verarbeitung in elektronischen Systemen.

Die Wiedergabe von Gebrauchsnamen, Handelsnamen, Warenbezeichnungen usw. in diesem Werk berechtigt auch ohne besondere Kennzeichnung nicht zu der Annahme, dass solche Namen im Sinne der Warenzeichen und Markenschutz-Gesetzgebung als frei zu betrachten wären und daher von jedermann benutzt werden dürften.

Lektorat: Ulrike M. Vetter

Gedruckt auf säurefreiem und chlorfrei gebleichtem Papier

Springer Gabler ist Teil der Fachverlagsgruppe Springer Science+Business Media.

www.springer-gabler.de 


\section{Vorwort}

Das Bundesministerium für Bildung und Forschung (BMBF) hat auf der Grundlage des BMBF-Programms „Arbeiten - Lernen - Kompetenzen entwickeln. Innovationsfähigkeit in einer modernen Arbeitswelt" Vorhaben zur Forschung und Entwicklung auf dem Gebiet „Innovationsfähigkeit im demografischen Wandel“ gefördert. Dabei ging es auch darum, die Innovationsfähigkeit in einer alternden und zahlenmäßig schrumpfenden Gesellschaft zu erhalten und durch die Verknüpfung von Arbeitsgestaltung mit Kompetenz-, Personalund Organisationsentwicklung zu stärken.

Mit dieser Ausrichtung setzte sich die Fokusgruppe „Altersheterogene Innovationsteams als Erfolgsfaktor des demografischen Wandels“ auseinander. In enger Kooperation von Wissenschaft und Wirtschaft sind in den Verbundprojekten der Fokusgruppe wichtige wissenschaftliche Erkenntnisse ermittelt sowie praxistaugliche Lösungen erarbeitet worden. Die Ergebnisse der beteiligten ArbeitsforscherInnen und UnternehmensvertreterInnen sind in diesem Band zusammengefasst. Ziel der Projekte ist der Erhalt und die Förderung der Innovationsfähigkeit der Beschäftigten, um damit die Wettbewerbsfähigkeit der Unternehmen und den Standort Deutschland zu stärken. Die Arbeiten der beteiligten Autoren sind auch wichtige Beiträge zur Forschungsagenda der Bundesregierung für den demografischen Wandel „Das Alter hat Zukunft“ und zur Demografiestrategie der Bundesregierung „Jedes Alter zählt“.

Die Projekte belegen eindeutig, dass das lange diskutierte Defizitmodell des Alterns nicht tragfähig ist, es wurde zu Recht längst abgelöst durch das Kompetenzmodell des Alterns. Individuen können sich durch die Anpassungs- und Änderungsfähigkeit an altersbedingte Beeinträchtigungen, veränderte Situationen und neue Voraussetzungen jederzeit anpassen, und diese Anpassung ist nicht zwangsläufig mit einer kontinuierlichen Abnahme der Leistungsfähigkeit verbunden, sondern ist gekennzeichnet durch ein hohes Potenzial an Lernfähigkeit und die zunehmende Ausprägung von Schlüsselkompetenzen. Damit ist auch sichtbar gemacht worden, dass der demografische Wandel keine Beschäftigtengruppen wie beispielsweise Ältere ausschließen darf und dass gerade deren Teilhabe wichtig ist. 
Die Projekte haben auch ein neues Orientierungswissen generiert, das belegt, wie wichtig es ist, im Kontext der Innovationsfähigkeit verstärkt den Fokus auf den gesamten Erwerbsverlauf zu legen und wie lernförderliche Erwerbsbiografien, das Miteinander unterschiedlicher Generationen und eine innovationsstärkende Unternehmenskultur zu gestalten sind.

Allen, die am Erfolg der geförderten Verbundprojekte und der Fokusgruppe mitgewirkt haben, möchte ich danken. Den Autorinnen und Autoren danke ich für die gelungene Aufbereitung der Projektaktivitäten und Projektergebnisse. Die vorliegende Arbeit verdient eine breite Leserschaft von denen, die aktiv mit der Innovationsfähigkeit im demografischen Wandels befasst sind: UnternehmerInnen, Führungskräfte, Betriebsräte, PersonalmanagerInnen, ArbeitsforscherInnen und ArbeitswissenschaftlerInnen sowie VertreterInnen von Arbeitgeberverbänden und Gewerkschaften und anderer intermediärer Institutionen.

Ilona Kopp

Projektträger im DLR

Arbeitsgestaltung und Dienstleistungen 


\section{Inhaltsverzeichnis}

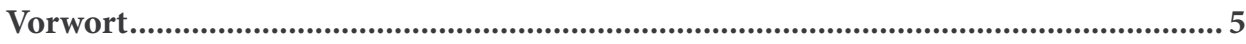

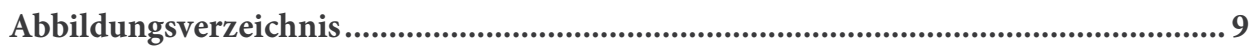

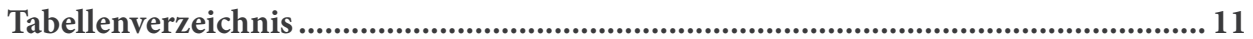

1. Die Bedeutung von Innovationskompetenz im demografischen Wandel als Voraussetzung zur Innovationsfähigkeit von Unternehmen ................................. 13 Thomas Langhoff

1.1 Erkenntnisse zur Veränderung kognitiver Funktionen ab dem mittleren Erwachsenenalter und ihr Einfluss auf Innovationskompetenz

1.2 Die Bedeutung von Erfahrungswissen und erfahrungsgeleitetem Arbeitshandeln für die Innovationsarbeit

1.3 Der Umgang mit dem Unplanbaren als Stärke älterer Arbeitnehmer in Innovationsprozessen.

1.4 Erkenntnisse zum Zusammenhang von Altern, Kreativität und Innovationskompetenz

1.5 Erkenntnisse zur Innovationskompetenz in altersheterogenen Arbeitsgruppen und vernetzten Strukturen

1.6 Die Fokusgruppe „Altersheterogene Innovationsteams als Erfolgsfaktor des demografischen Wandels“

2. Innovationskompetenz in der Praxis -

Instrumente und betriebliche Gestaltungslösungen 43

Thomas Langhoff, Stefan Schröder 
2.1 Arbeitgeberattraktivität im demografischen Wandel -

Entwicklungspotenziale und Handlungsfelder

Arne Brand, Bernd Lachmann, André Schubert

2.2 Demografiefestes Personalmanagement - Arbeitsfähigkeit,

Qualifizierung und alter(n)sgerechter Personaleinsatz.

Kai Rosetti, André Schubert, Alexander Walter

2.3 Innovation auf mehreren betrieblichen Ebenen steigern -

Ein branchenunabhängiges Konzept für alle Altersgruppen

Kristina Küper, Theresa Myskovszky von Myrow, Christian Ahlfeld

2.4 Training von Führungskompetenzen zur Steigerung der Innovation und Produktivität in altersgemischten Teams

Laura Bröker, Wladislaw Rivkin, Julia Günnewig

2.5 Gesundheit - Basis kreativer Leistungen in

Forschung und Entwicklung.

Birgit Ottensmeier, Alexander Knickmeier, Christoph Kuth

2.6 Altersdiversität und Leistung in Innovationsteams.

Madlen Hiller, Anne Köhn, Philipp Przybysz

2.7 Personen und Prozesse in Innovationsprojekten:

Simulation und empirische Befunde.

Martin Ratzmann, Sebastian Terstegen, Reinhard Weiß

2.8 Fabrikplanung und demografischer Wandel am Beispiel

der Firma Fahrion Engineering GmbH \& Co. KG

Jens Fahrion

2.9 Ältere Beschäftigte als Innovationsexperten

Eckhard Heidling, Vera Kahlenberg, Bernhard Ludwig, Judith Neumer

3. Innovationskompetenz im demografischen Wandel:

Zusammenfassung der Erkenntnisse und weiterer Forschungsbedarf. 199

Thomas Langhoff, Stefan Schröder 


\section{Abbildungsverzeichnis}

Abb. 1.1 Unterschiede in der Persönlichkeit von Kreativen und Innovatoren (nach Schuler \& Görlich, 2007).

Abb. 1.2 Dimensionen des Teamklimas und Phasen des Innovationsprozesses im Vergleich (Langhoff, 2011a, zitiert nach Packebusch, 2010); (Basadur 1994); West, 1990)

Abb. 1.3 Gegenüberstellung von arbeitsorientiertem und konventionellem Innovationsverständnis ( Langhoff, 2011a)

Abb. 2.1 Beispielhafte Befragungsergebnisse aus den Analyseworkshops. .51

Abb. 2.2 Einflussfaktoren auf die Leistungsfähigkeit über das Lebensalter hinweg (BDA 2013: 5, in Anlehnung an Buck et al., 2002).

Abb. 2.3 Auszug aus einem ausgefüllten Ergo ${ }^{{ }^{\mathrm{PE}}}$-Erfassungsbogen aus der Dimension „Arbeitsumgebung“ (fiktives Beispiel). .66

Abb. 2.4 Die Ergo ${ }^{\mathrm{PE}}$-Belastungsampel 66

Abb. 2.5 Die Ergo ${ }^{\mathrm{PE}}$-Belastungskarte: Das Belastungsniveau eines Arbeitsplatzes im Überblick (fiktives Beispiel)

Abb. 2.6 Die Ergo. ${ }^{\mathrm{PE}}$-QualiMatrix: Das Belastungsniveau von Arbeitsplätzen und die Qualifikationsstruktur der Beschäftigten im Überblick (fiktives Beispiel) ....69

Abb. 2.7 Das Ergo. ${ }^{\text {PE }}$-Formular für die ermittelten Maßnahmen: Bewertung nach Realisierbarkeit und Wirksamkeit im Überblick (fiktives Beispiel)

Abb. 2.8 Integratives Interventionskonzept zur Innovationssteigerung auf mehreren betrieblichen Ebenen (nach Küper et. al, im Druck) 78

Abb. 2.9 Beschäftigte der Deutschen Gasrußwerke GmbH \& Co. beim kognitiven Training 80 
Abb. 2.10 Sieben Kernkompetenzen des Stress-Management-Trainings (nach Berking, 2010) 81

Abb. 2.11 Spannungsfeld unternehmensgetriebener Innovationsprozesse ......................86

Abb. 2.12 Gruppenübung „Strategische Verhaltensweisen von Vorgesetzten“.................97

Abb. 2.13 Ranking berufsbezogener Motive (Grube, 2009)............................................. 100

Abb. 2.14 Facetten von Handlungskompetenz .................................................................... 100

Abb. 2.15 Reifegradmodell von Hersey \& Blanchard (1982) ........................................... 101

Abb. 2.16 Erwartete Effekte des Servant Leadership Trainings .......................................107

Abb. 2.17 Individuelle und organisationale Ressourcen für Kreativität und Innovationen in F\&E (in Anlehnung an Amabile 1997)

Abb. 2.18 Anzahl der Beschäftigten nach Ausprägung von Vitaler Erschöpfung in fünf Altersgruppen, $\mathrm{N}=424$

Abb. 2.19 Änderungswünsche in gesundheitsbezogenen Lebensbereichen nach Altersgruppen.

Abb. 2.20 Bedingungskreislauf zwischen Arbeitsverhältnissen, individuellem Selbstbezug, Verhalten und Beziehungsgestaltung....

Abb. 2.21 Abgewandeltes IPO-Modell nach Gladstein (1984)

Abb. 2.22 Der Interaktionseffekt der Altersdiversität und des integrierten Konflikthandhabungsstils auf die Innovation insgesamt.

Abb. 2.23 Ausschnitt des grafischen K3-Prozessmodells des Innovationsprojektes der EGT. Rechts: Übersicht über das gesamte Prozessmodell...

Abb. 2.24 Histogramm der simulierten Projektlaufzeit 146

Abb. 2.25 Input-Process-Output-Rahmenmodell zur Analyse von Gruppenverhalten und -leistung in Anlehnung an Hackman (1987)

Abb. 2.26 Projektlaufzeit in Abhängigkeit von Teamgeist sowie Effektivität und Effizienz

Abb. 2.27 Relevante Marker der Teamarbeit. Der Bereich zwischen den gepunkteten Linien weist auf den durchschnittlichen Wertebereich innerhalb der empirischen Studie hin (Mittelwert +/- eine Standardabweichung). Die durchgezogene Linie zeigt die Ausprägung der Marker innerhalb der Fallstudie auf.

Abb. 2.28 Professionelles Arbeitshandeln als Verschränkung subjektivierender und objektivierender Merkmale (vgl. Böhle, 2004; Böhle et al., 2012) 166

Abb. 3.1 Erfolgsfaktoren altersheterogener Innovationsteams (weiterentwickelt nach Wegge et al., 2011) 201 


\section{Tabellenverzeichnis}

Tab. 1.1 Gegenüberstellung des früheren Ansatzes des Defizitmodells vom Altern mit dem heute gültigen Kompetenzmodell des Alterns........................... 16

Tab. 1.2 Promotorenmodell im Innovationsprozess (nach Hauschildt, 2004)................22

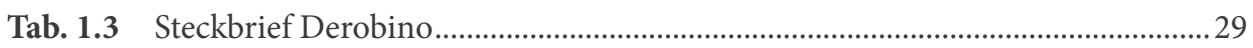

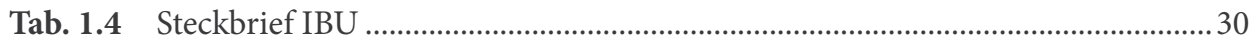

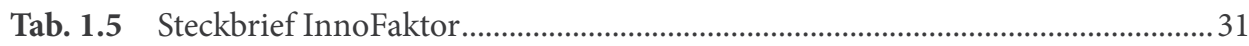

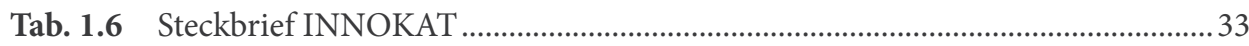

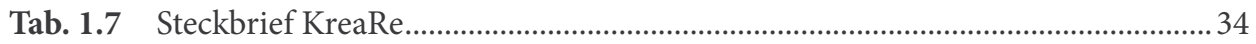

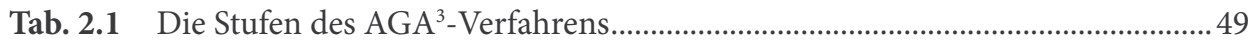

Tab. 2.2 Moderationsfragen für die Plenumsdiskussion und Kleingruppenarbeit .........52

Tab. 2.3 Leitfaden für die Erarbeitung von konkreten Handlungsmaßnahmen.............54

Tab. 2.4 Methode zur Priorisierung von Handlungsempfehlungen

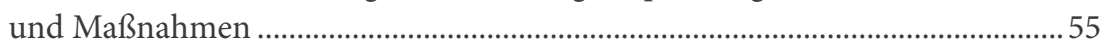

Tab. 2.5 Ergebnisse der Analyseworkshops bei KIND Hörgeräte.......................................57

Tab. 2.6 Ergebnisse der Kleingruppenarbeit in dem Zusammenführungsworkshop ....58

Tab. 2.7 Dimensionen des Servant Leadership nach Liden et al. (2008) .........................91

Tab. 2.8 Übung „Die Abschiedsrede“ nach Küper et al. (in Druck) ..................................99

Tab. 2.9 Gruppenübung „Strategische Verhaltensweisen von Vorgesetzten“..................97

Tab. 2.10 Ranking berufsbezogener Motive (Grube, 2009) ................................................99

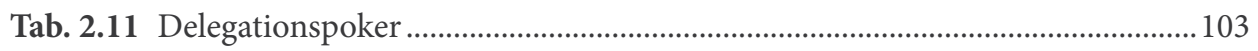

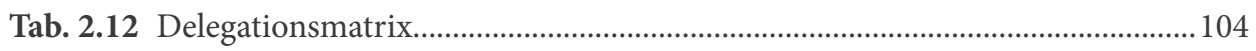

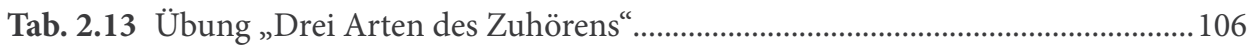


Tab. 2.14 Stressbewältigung durch Achtsamkeit -

Acht-Wochen-Inhouse-Trainingskurs 121

Tab. 2.15 Prognosen der Projektlaufzeit................................................................................. 152

Tab. 2.16 Innovationskompetenzen altersneutral/altersspezifisch.................................... 185 